10.1590/S1414-40772018000200015

Este é um artigo publicado em acesso aberto sob uma licença Creative Commons https://creativecommons.org/licenses/by-nc/4.0/

\title{
Retos de la educación superior en un contexto de incertidumbre y crisis global
}

\author{
Francisco Lopez Segrera ${ }^{1}$
}

Resumen: Las sintéticas reflexiones de este texto tienen como objetivo identificar los retos que enfrentan los sistemas de educación superior y las universidades de América Latina y el Caribe, ante los posibles escenarios que nos aguardan en un momento de crisis e incertidumbre, tanto a nivel mundial, como en lo que respecta a nuestra región. También ofrece recomendaciones con el fin de construir alternativas que contribuyan a que prevalezcan los mejores escenarios posibles.

Palabras clave: Educación Superior; Crisis global y incertidumbre; América Latina y Caribe.

\section{Challenges of higher education in a context of uncertainty and global crisis}

Abstract: The Synthetic reflections of this text have as aim to identify the challenges that face the systems of higher education and the universities in Latin America and the Caribbean, concerning the possible scenarios emerging in a moment of crisis and uncertainty, worldwide, and in our region. Additionally, it offers recommendations in order to construct alternatives that can foster the best possible scenarios.

Key words: Higher education; Global crisis and uncertainty; Latin America and the Caribbean.

\section{Desafios do ensino superior em um contexto de incerteza e crise global}

Resumo: As reflexões sintéticas deste texto visam identificar os desafios enfrentados pelos sistemas de ensino superior e universidades da América Latina e do Caribe, diante dos possíveis cenários que nos esperam em um momento de crise e incerteza, tanto global como globalmente. em relação à nossa região. Também oferece recomendações para construir alternativas que contribuam para prevalecer os melhores cenários possíveis.

Palavras-chave: Educação superior; Crise global e incerteza; América Latina e Caribe. 


\section{Posibles escenarios en un contexto de incertidumbre y crisis}

Durante el año 2016 hemos contemplado la emergencia de varios cisnes negros, a la manera de sucesos imprevistos de alto impacto: la tensión entre la UE y Rusia, entre EE.UU. y Rusia, entre la OTAN y Rusia; los conflictos bélicos en el Próximo Oriente, la presencia de DAESH, la guerra en Siria y la migración masiva hacia Europa; el Brexit, qué afectará las relaciones de las universidades inglesas con las europeas y pudiera dar lugar a una emigración de académicos hacia otros países; la victoria de Trump en EE.UU. y los rasgos de extrema derecha de su gobierno; la crisis de los procesos pos-neoliberales de izquierda en América Latina y el Caribe y el ascenso de una nueva derecha; y las consecuencias de la baja de los precios del petróleo y las materias primas. ${ }^{1}$

Las universidades enfrentan una de las épocas más interesantes, inciertas y complejas, pues la globalización implica la posibilidad de aprovechar oportunidades importantes, pero también desafíos y problemas serios con relación al futuro, al cuestionarse el ideal de lo público y del bien común. Los valores tradicionales de la Universidad siguen siendo válidos (la autonomía, la libertad de cátedra, la investigación, el trabajo de los estudiantes, la evaluación), pero son valores amenazados en el contexto de la globalización. ${ }^{2}$

Por otra parte, transformar una universidad para intentar llevarla a la categoría de "clase mundial" con iniciativas de excelencia, implica políticas nacionales de mediano y largo plazo y un sólido financiamiento. ¿Es la solución, como ha hecho China, plantearse un cronograma para que algunas de sus universidades alcancen la "clase mundial"??

Estamos viviendo en un sistema-mundo caótico, donde países de envergadura como EE.UU. y Reino Unido, entre otros, plantean la vuelta al proteccionismo y en el cual, si bien la pobreza se ha reducido ligeramente, la desigualdad aumenta aceleradamente.

1 Tras la victoria de Trump, algunos analistas opinan que la era de la globalización y el neoliberalismo toca a su fin y comienza la del neonacionalismo. ¿Qué consecuencias podría tener esto para América Latina y para la educación superior? Han existido muchos criterios sobre el futuro de las universidades, desde el de Drucker que anunció su desaparición hace 3 décadas, hasta el de Bill Gates en 2014, cuando afirmó que cursos virtuales de alta calidad podrían reemplazar a los cursos presenciales y alentando a las universidades públicas a adoptar procedimientos de control y eficiencia propios de las universidades for-profit.

2 La revolución micro-electrónica, la sociedad de la información y el conocimiento y la vertiginosa expansión de la educación superior, han implicado desde los 90s una urgencia por profundizar en los estudios prospectivos.

Los Informes de UNESCO como el Informe Delors y los de las Conferencias Mundiales de Educación Superior. Los Informes de Naciones Unidos y en especial las Metas del Milenio sustituidas en 2015 por los nuevos Objetivos del Desarrollo Sostenible. Esfuerzos como los del Proyecto Milenio y su Estado del Futuro y el que desarrolló la Red Latinoamericana de Estudios Prospectivos. Los informes de CEPAL. Los estudios prospectivos llevados a cabo por la OCDE y los Informes de la Global University Network for Innovation (GUNI), entre otros, nos ofrecen una notable masa crítica de conocimiento acerca de los retos de la educación superior en un contexto de crisis e incertidumbre global.

3 El reciente" Informe de UNESCO sobre la Ciencia: Hacia el 2030" demuestra que China ocupa el segundo lugar en el gasto de I+D a nivel mundial con un $20 \%$ - solo superado por un $28 \%$ en EE.UU- y por encima de la Unión Europea (19\%) y Japón (10\%). 
En este contexto de incertidumbre y crisis global la buena noticia es que si bien sabemos que no vamos ineluctablemente hacia el progreso, también estamos conscientes de que la construcción del futuro está en nuestras manos. Ya no somos rehenes de la teleología hegeliana, ni del positivismo. No existe el futuro pre-determinado como señaló Prigogine (1996) y tenemos las herramientas de la prospectiva y del pensamiento complejo de Morin (2004) para construir un futuro alternativo al caos y a la acelerada destrucción del medio ambiente. Según Morin (2004, p.77, traducción nuestra) "el pensamiento que enfrenta la incertidumbre puede esclarecer las estrategias en un mundo incierto".

En 2013, el politólogo Immanuel Wallerstein se refería a 3 imponderables que podrían afectar cualquier proceso de transición sistémica hacia otro sistema-mundo posterior al capitalismo actual: el cambio climático, las pandemias y la guerra nuclear. Según él, el sistema-mundo capitalista está en una crisis estructural que conduce a una lucha política a escala mundial en que pudieran prevalecer - dentro de 20 o 40 años - uno de los dos escenarios siguientes (WALLERSTEIN, 2013):

a) Uno que tenga por resultado un sistema no capitalista, que retenga todos los peores rasgos del capitalismo (las jerarquías, la explotación y la polarización).

b) Otro que siente las bases para un sistema basado en una relativa democratización y un relativo igualitarismo, un tipo de sistema que aún no ha existido.

Para este autor, antes del 2020 se producirá una proliferación nuclear a escala significativa y seremos testigos tanto de un proteccionismo como de una deflación mundial generalizada.

Entre 2016 y el 2030 visualizamos los siguientes escenarios:

a) Hegemonía Imperial. El actual.

b) Ingobernabilidad Global. Podría ser el resultado de un cuestionamiento de la gobernabilidad imperial por potencias emergentes.

c) Democracia Internacional Consensuada. Sería el resultado de la victoria de las negociaciones a nivel global y de transformaciones cualitativas esenciales en el sistema mundo capitalista actual.

Otra forma de representar los posibles escenarios es en una tabla con 4 casillas para los diversos modelos de futuro posibles ${ }^{4}$ :

\footnotetext{
4 Ver en el País, Negocios, 27/11/2016 el artículo de Antón Costas “Alternativa al cosmopolitismo y al populismo". He tomado de este artículo algunos elementos para la elaboración de esta tabla, pero también tengo diferencias con el análisis original de Costas.
} 


\begin{tabular}{|c|c|}
\hline $\begin{array}{l}\text { LIBERTAD TOTAL DE CAPITALES Y DE } \\
\text { COMERCIO DE LA GLOBALIZACIÓN } \\
\text { ACTUAL Y SOCIEDAD DE TIPO } \\
\text { INDIVIDUALISTA EXTREMO. ES } \\
\text { ACTUALMENTE EL MODELO } \\
\text { HEGEMÓNICO A NIVEL MUNDIAL QUE } \\
\text { SUSTENTA EL CAPITAL FINANCIERO Y } \\
\text { EL TRANSNACIONAL Y LAS CLASES } \\
\text { POLÍTICAS DOMINANTES. }\end{array}$ & $\begin{array}{l}\text { COMBINA UNA GLOBALIZACIÓN } \\
\text { RAZONABLE CON UN NUEVO CONTRATO } \\
\text { SOCIAL QUE COMPENSE A LOS } \\
\text { PERDEDORES DE LA GLOBALIZACIÓN, } \\
\text { REPARTE MEJOR LOS BENEFICIOS DEL } \\
\text { CRECIMIENTO REDUCIENDO A NIVELES } \\
\text { CADA VEZ MENORES LA POBREZA Y LA } \\
\text { DESIGUALDAD Y AMPLIANDO LA } \\
\text { PARTICIPACIÓN POLÍTICA REAL Y LA } \\
\text { DEMOCRACIA. ES EL QUE HAN } \\
\text { INTENTADO DESARROLLAR LOS } \\
\text { GOBIERNOS POSNEOLIBERALES EN } \\
\text { AMÉRICA LATINA Y EL CARIBE Y HACIA } \\
\text { EL QUE PARECEN DIRIGIRSE POR } \\
\text { DIVERSAS VÍAS PAÍSES QUE } \\
\text { DESARROLLAN EXPERIENCIAS } \\
\text { SOCIALISTAS COMO CHINA, CUBA Y } \\
\text { VIETNAM. TAMBIÉN EL “ESTADO DE } \\
\text { BIENESTAR DE EUROPA” SE ACERCÓ A } \\
\text { ESTE MODELO HASTA QUE COMENZÓ A } \\
\text { DEMOLERLO LA GLOBALIZACIÓN } \\
\text { NEOLIBERAL. SECTORES DE LOS } \\
\text { PARTIDOS SOCIALISTAS EUROPEOS Y DE } \\
\text { LOS RECIENTES POPULISMOS DE } \\
\text { IZQUIERDA ABOGAN POR ESTE MODELO. }\end{array}$ \\
\hline $\begin{array}{l}\text { RETORNO AL PROTECCIONISMO Y AL } \\
\text { NACIONALISMO ECONÓMICO CON UNA } \\
\text { VISIÓN INDIVIDUALISTA, RACISTA Y } \\
\text { XENÓFOBA DE LA SOCIEDAD. ES } \\
\text { PROPIA DEL POPULISMO DE DERECHA } \\
\text { DE TRUMP, DE LOS AUSPICIADORES DEL } \\
\text { BREXIT Y DE MARIE LEPEN, ENTRE } \\
\text { OTROS. }\end{array}$ & $\begin{array}{l}\text { PROTECCIONISMO Y NACIONALISMO } \\
\text { ECONÓMICO. ES LA QUE OFRECEN } \\
\text { ALGUNOS POPULISMOS NACIONALISTAS } \\
\text { DE IZQUIERDA Y BUENA PARTE DE LOS } \\
\text { MOVIMIENTOS INDEPENDENTISTAS. }\end{array}$ \\
\hline
\end{tabular}

Fuente: Elaboración propria, com base en Costas (2016)

Estamos ante la siguiente alternativa: un mundo cada vez más desigual y contaminado o bien más solidario, igualitario, democrático y sostenible. ${ }^{5}$

\footnotetext{
5 Pero para que triunfe esta alternativa solidaria y democrática es necesario llevar a cabo políticas que impliquen: modificar un estilo de vida consumista; establecer políticas sociales y de acción afirmativa que brinden igualdad de oportunidades, eliminen la pobreza e impidan todas las formas de discriminación, reduciendo a un mínimo las desigualdades sociales y económicas mediante un adecuado sistema impositivo; lograr que los países desarrollados cumplan el compromiso que hicieron de aportar el 0,7\% de su PNB como asistencia al desarrollo; adoptar políticas efectivas de erradicación de la pobreza; apoyar los proyectos de ciudades inteligentes y la adecuada planificación urbana; dar prioridad al transporte público; gravar con altos impuestos los autos de alta cilindrada y los vehículos más contaminantes; priorizar inversiones públicas que tiendan a solucionar el desempleo y en especial el juvenil; establecer y/endurecer legislaciones y políticas adecuadas contra la violencia de género; facilitar la paridad tanto en los puestos de trabajo como en los de dirección de hombres y mujeres; llevar a cabo políticas de acción afirmativa con relación a las mujeres, la igualdad cívica y civil, y hacia todos aquellos que por razones de sexo, raza u otras sufren discriminación y exclusión social; educar a los jóvenes y movilizar a los gobiernos en la lucha contra el tráfico de drogas y la dependencia, sin dejar de valorar la legalización en algunos casos; brindar un mayor apoyo por parte de los gobiernos al Programa de Naciones Unidas para el Medio Ambiente y establecer medidas punitivas en el ámbito de Naciones Unidas contra aquellos países y empresas que no cumplan con las medidas que evitan que prosiga la degradación del medio ambiente y el cambio climático; perfeccionar las políticas de preservación de los recursos hídricos y todas aquellas que contribuyan a preservar la cantidad y calidad del agua; adoptar legislaciones y políticas que impidan que la desertificación y deforestación siga avanzando; desarrollar iniciativas globales de seguridad alimentaria teniendo en cuenta factores geográficos, demográficos y adoptando legislaciones que regulen los altos precios de los alimentos básicos; aplicar la biotecnología en todas aquellas áreas que sea posible siguiendo normas éticas; desarrollar la educación permanente para todos para toda la vida a nivel mundial; vincular
} 
"La universidad tiene responsabilidades sobre el modelo de desarrollo de la sociedad global. Ese modelo debe ser construido en una dirección distinta a la que hoy se impone como hegemónico" (DIAS SOBRINHO, 2015, p. 596).

La crisis de la representación del futuro como crecimiento exponencial indefinido a nivel planetario y de las etapas del crecimiento económico como una marcha de todos hacia una felicidad compartida, han quebrantado en el Sur - y también en el Norte - la certidumbre de la civilización occidental como modelo a imitar y también en modelos socialistas alternativos que se derrumbaron.

El futuro se presenta abierto y diverso. Estamos ante una amplia gama de posible futuro, ante una bifurcación de senderos que nos ofrece una ramificación de posibles caminos de desarrollo. No se trata de un único porvenir ineluctable, sino de los "futuribles", de los futuros posibles que podemos construir. Es necesario cambiar radicalmente nuestro modelo consumista y abandonar la ideología productivista que ha prevalecido tanto en los sistemas socialistas que se derrumbaron como en el capitalismo desarrollado. Con estos modelos productivistas no es posible el desarrollo sostenible.

\section{Retos que enfrenta la educación superior en este contexto de incertidumbre y crisis global}

Los retos que deberá enfrentar la educación superior estarán condicionados por el escenario de sistema-mundo que prevalezca. Pero es importante destacar que con nuestras recomendaciones y acciones en el ámbito universitario podemos contribuir a construir el mejor escenario, esto es, el escenario apuesta. ${ }^{6}$

\footnotetext{
las formas más sofisticadas del saber de la sociedad del conocimiento con formas ancestrales de saber propias de las comunidades indígenas; lograr que las autopistas de la información e internet no den lugar a una brecha de información entre info-ricos e info-pobres; favorecer el desarrollo de energías no contaminantes y penalizar con altos impuestos las contaminantes; promover el acceso al libro y la lectura y celebrar concursos que estimulen la creación literaria y artística; velar por aquellas culturas y lenguas que están hoy amenazadas por la urbanización acelerada y otros factores; erradicar todas las formas de violencia y de violación de los derechos humanos; y lograr desarrollar una cultura de paz a nivel mundial que implique la solución negociada de los conflictos sin hacer uso de las armas. Si las clases políticas no quieren seguir siendo percibidas cada vez más como cómplices y subordinados de los círculos financieros deben adoptar medidas de regulación de los mercados tales como: eliminar los paraísos fiscales con legislaciones adecuadas; los bancos rescatados deben pagar un salario a sus empleados no mayor al de los empleados públicos; los políticos no cobrarán jubilación si pasan a trabajar en el sector privado; los directivos de las empresas o bancos que quiebren no recibirán ninguna remuneración y deberán afrontar personalmente estas pérdidas.

6 Tenemos pendiente en nuestra Agenda de ALC la necesidad de una transformación profunda en las formas de transmitir, producir y aplicar el conocimiento con el fin de formar una ciudadanía del siglo XXI y alcanzar sistemas productivos de competitividad internacional. Esto debe lograrse en el marco de un proceso de consolidación de la democracia en ALC, de disminución de la pobreza y la indigencia, pero de aumento de la desigualdad, en una coyuntura de cambio vertiginoso en los sistemas de educación superior a nivel mundial y con una idea de la universidad en que se combinan el financiamiento público y el privado y las funciones docentes, de investigación y del tercer sector (servicios, extensión, difusión, consultorías,...).
} 
Si predomina el escenario de un sistema mundo cada vez más desigual y contaminado y con ingobernabilidad global, podrían prevalecer en la educación superior escenarios tales como:

a) Mercado libre en el que imperan las fuerzas del mercado.

b) Educación Superior Inc., en que las IES compiten globalmente para proveer servicios educativos y de investigación sobre bases comerciales y no sociales.

c) Predominio de la competitividad privatizadora versus la cooperación solidaria.

d) En América Latina y el Caribe hoy prevalece el escenario de Globalización con subordinación y mercado actual "indefinido". La tendencia es a la privatización creciente de la educación superior. Si predomina el escenario de ingobernabilidad global el escenario actual podría evolucionar hacia un escenario de Globalización con subordinación y mercado regulado por acuerdos comerciales (como GATS). Esto implicaría la desnacionalización de la educación superior ante la llegada indiscriminada de proveedores con ánimo de lucro.

Si predomina el escenario de "democracia internacional consensuada", que implique paz mundial, y políticas que garanticen la sostenibilidad del planeta, así como la reducción acelerada de la pobreza y la desigualdad, pudieran prevalecer en la educación superior los siguientes escenarios:

a) Universidades emprendedoras e innovadoras. Actúan con autonomía ante varias fuentes de financiamiento.

b) Red global de instituciones. Orientadas a la cooperación internacional y a la solución de los problemas nacionales y locales con una visión local que aporta a lo global.

c) Redes abiertas: la educación superior muestra un alto grado de internacionalización que implica vínculos entre diversas redes y se basa más en la cooperación que en la competencia. Es uno de los escenarios que propone la OCDE (2008), similar al anterior.

d) Sirviendo a las comunidades locales. La educación superior es administrada y financiada por el Estado y se enfoca la docencia y la investigación a los problemas nacionales y locales.

e) En el caso de ALC pudiera extenderse y perfeccionarse un escenario que ha venido emergiendo, similar al anterior, de Globalización sin subordinación y con cooperación independiente. Un creciente número de países - Cuba, Venezuela, Bolivia, Ecuador, Uruguay...- establecen acuerdos de cooperación en el ámbito universitario sin subordinarse a los acuerdos comerciales del GATS.

\section{Las tendencias mundiales y regionales de la educación superior como retos}

De acuerdo con López Segrera (2016), las tendencias mundiales y regionales de la educación superior son: 
a) Masificación: la tasa bruta de matrícula (TBM) a nivel mundial pasó de 13 millones de estudiantes en 1960 a 207 millones en 2014 (UNESCO.UIS, 2017).

b) La educación se ha hecho permanente.

c) La aparición de las TIC implica que el equipamiento de una universidad es más costoso.

d) El incremento vertiginoso de la movilidad académica internacional y la "fuga de cerebros". Los estudiantes internacionales pasaron de 2.5 millones en el 2004 a cerca de 5 millones en 2016.

e) El financiamiento de la educación superior descansa en recursos públicos y privados y estos últimos aumentan su proporción.

f) La crisis de la profesión académica.

g) ¿Cómo ser innovadores construyendo igualdad y sostenibilidad?

\section{4 iA transitado realmente la educación superior de América Latina y el Caribe de la} educación superior de masas a la universalización de la educación superior?

De acuerdo con la clasificación de Martin Trow (2005), los sistemas de educación superior en América Latina y el Caribe ya no tienen ningún caso con educación superior de élite (tasa de matrícula de menos del 15\%), sino que transitan de la educación superior de masas (entre el 15 y el 50\% de tasa de matrícula) a la universalización con tasas de matrícula superiores al $50 \%$ en algunos casos, aunque lo que predomina aún es la educación superior de masas, por debajo del 50\% de tasa de matrícula (TROW, 2005; UNESCO.UIS, 2017).

\section{Tabla 1}

\begin{tabular}{|c|l|c|}
\hline REGIÓN & \multicolumn{1}{|c|}{ PAÍS } & TBM/GER \\
\hline \multirow{4}{*}{$\begin{array}{c}\text { AMÉRICA } \\
\text { LATINA Y EL }\end{array}$} & CHILE & 89 \\
\cline { 2 - 3 } CARIBE & ARGENTINA & 83 \\
\cline { 2 - 3 } & COLOMBIA & 56 \\
\cline { 2 - 3 } & BRASIL & 49 \\
\cline { 2 - 3 } & MÚXICO & 36 \\
\cline { 2 - 3 } & JAMAICA & 30 \\
\cline { 2 - 3 } & HONDURAS & 27 \\
\cline { 2 - 3 } & GUATEMALA & 21 \\
\hline
\end{tabular}

Si bien el promedio de la tasa de matrícula a nivel regional se incrementó del 17\% a principios de los años 90s al 45\% en 2017, esta cifra sigue estando muy por debajo de las tasas de matrícula de los países desarrollados. Además de este bajo monto en las tasas de 
matrícula las tasas de deserción son altas y las de graduaciones bajas. A esto se añade la inequidad en al acceso a la educación superior (Ver Gráfico 1)

Tasa de participación de los estudiantes del quintil más pobre en la educación terciaria, 2014 (en porcentaje).

\section{Gráfico 1}

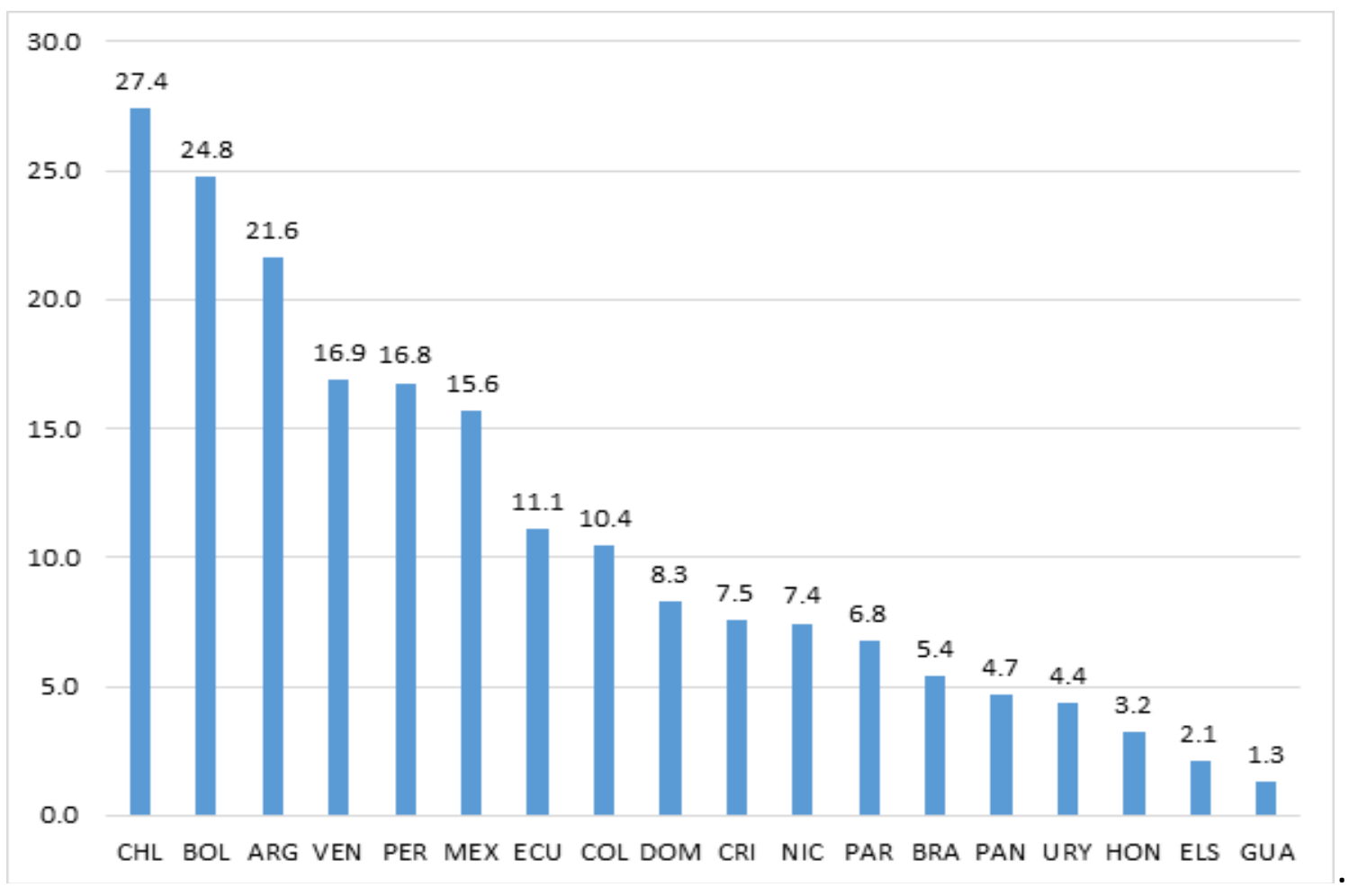

Fuente: BRUNNER, J. J. Educación Superior Iberoamericana. Santiago: Cinda, 2016.

Por otra parte, mucho de este crecimiento en la matrícula, además de deficitario cuantitativamente, lo es cualitativamente. Mucho de este aumento en las TBM es el resultado de la aparición de instituciones privadas de absorción de demanda de baja calidad. Aparecen además proveedores internacionales de educación superior privada sin un adecuado control de la calidad de los estudios que ofrecen.

Participación de la matrícula privada en el total de la matrícula de educación terciaria por país, 2013/2014 (en porcentaje). 


\section{Gráfico 2}

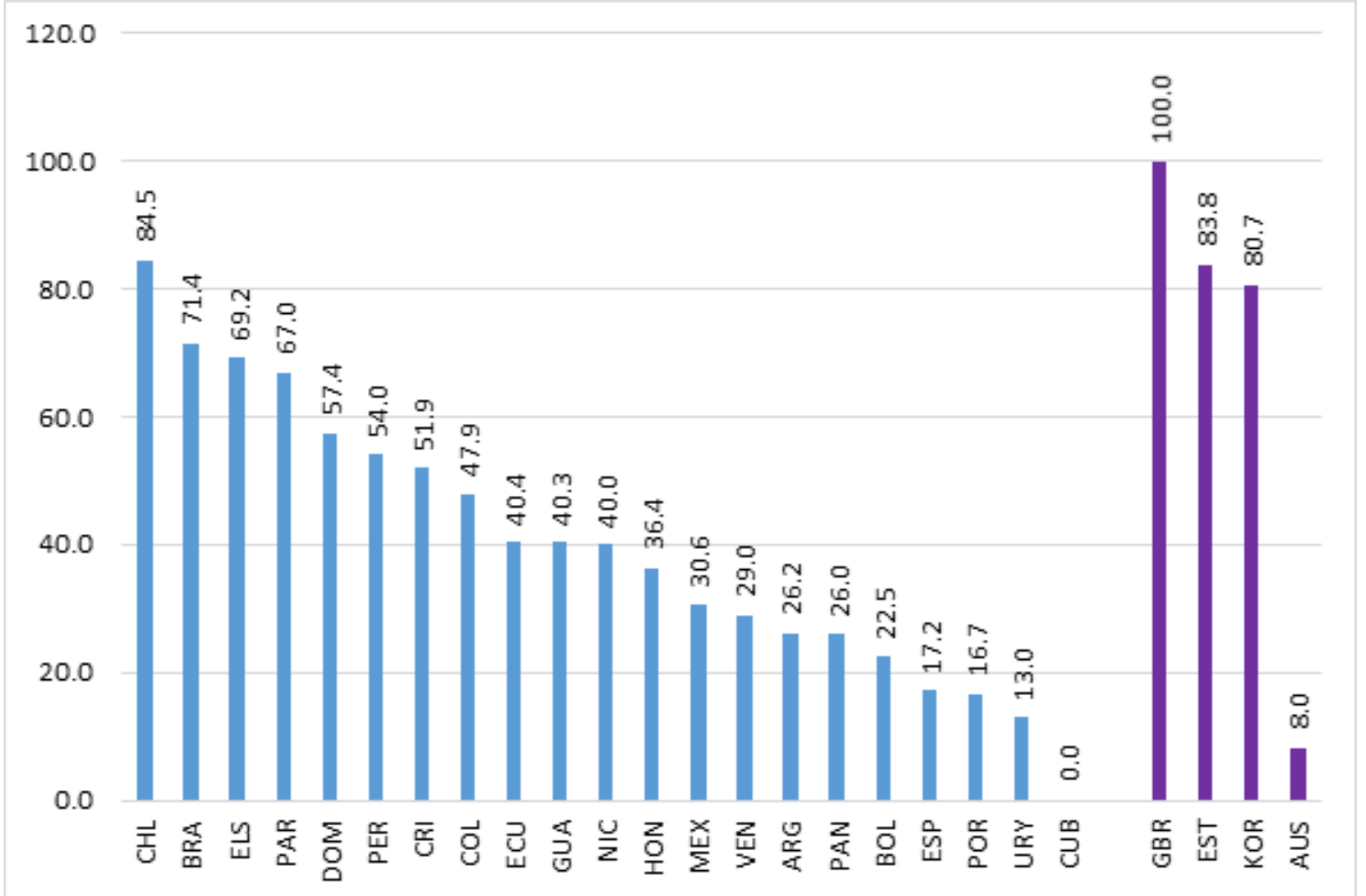

Fuente: BRUNNER, J. J. Educación Superior Iberoamericana. Santiago: Cinda, 2016.

Por otra parte, si bien los procesos académicos se han perfeccionado en las grandes universidades públicas de los diversos países y en algunas privadas de calidad, aún en muchos aspectos no se ha superado la tendencia a la profesionalización propia del modelo napoleónico. Si bien se han incrementado los posgrados de calidad - concentrados en Argentina, Brasil y México -, los indicadores de la región en Ciencia y Tecnología siguen estando a la zaga y solo por delante de los países de África y de algunos países pobres de Asia.

Se han producido avances en lo que respecta a la internacionalización de la educación superior, pero sigue siendo una de las regiones menos atractivas para los estudiantes internacionales. Solo un $2 \%$ de ellos acuden a cursar estudios en países latinoamericanos. España, Cuba y Brasil están a la vanguardia en este ámbito.

Número de estudiantes extranjeros que cursan estudios superiores dentro de un país, 2013 (Gráfico 3). 


\section{Gráfico 3}

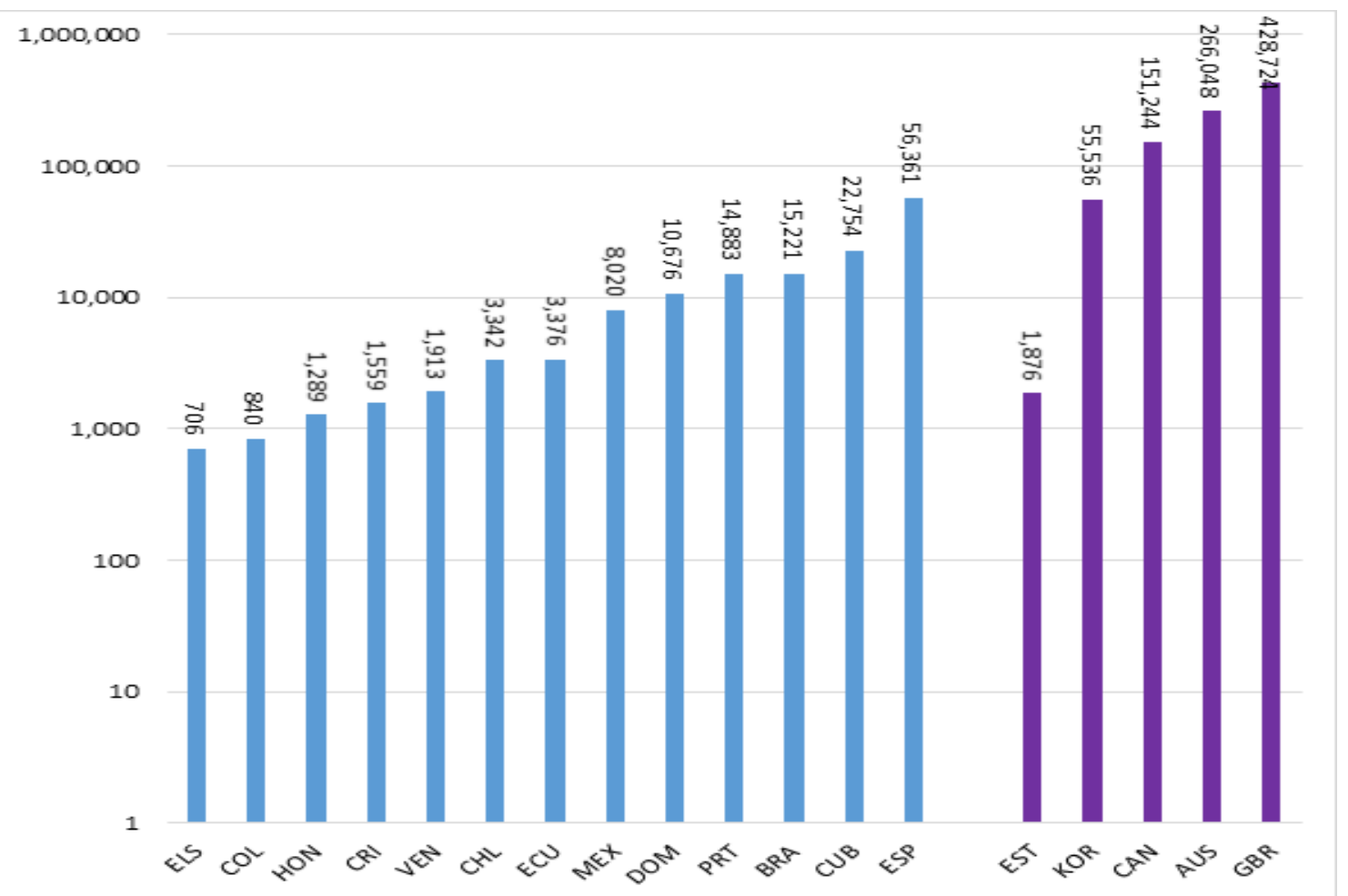

Fuente: BRUNNER, J. J. Educación Superior Iberoamericana. Santiago: Cinda, 2016.

\section{Retos claves que debemos enfrentar en los sistemas de educación superior y en las}

\section{universidades:}

a) El Reto de la Inequidad.

b) El Reto del formalismo y la burocratización en los procesos de evaluación y acreditación.

c) El Reto del mimetismo de las "World Class Universities".

d) El Reto de privilegiar los indicadores productivistas y de crecimiento económico (muchas veces no sostenible) en detrimento de indicadores que midan valores claves para la equidad, la armonía social y la sostenibilidad del medio ambiente.

e) El Reto de la "corrupción académica".

f) El Reto de la acreditación internacional.

g) El Reto de Innovar en lo local, nacional y mundial partiendo del estado del arte del conocimiento a nivel global.

Pienso que instituciones como UNESCO y GUNI, instituciones como IESALCUNESCO y sus redes, y otras redes y universidades de excelencia, pueden construir interactivamente una importante masa crítica de conocimiento al respecto y en esa dirección se está avanzando. 


\section{Conclusiones}

Estas reflexiones nos llevan a la siguiente consideración: ¿Cuál escenario consideramos el ideal para enfrentar los retos actuales en una coyuntura de crisis e incertidumbre a nivel mundial? ¿Por qué razones? ¿Qué alternativas de políticas debemos apoyar para impulsar el mejor escenario posible a nivel nacional, regional y global? ¿Acaso no es necesario construir un nuevo escenario más deseable que los que existen o se han formulado? ¿Cuál sería el escenario que contribuiría a qué nuestras universidades latinoamericanas den respuesta adecuada a las expectativas nacionales de aporte al desarrollo, al tiempo que satisfacen los estándares internacionales? ¿Qué plan estratégico y acciones hay que desarrollar al respecto? También podríamos plantearnos lo siguiente: ¿no será que en el futuro coexistirán escenarios diversos de carácter heterogéneo de acuerdo a las características diversas de naciones y regiones? ¿Hay alguna correlación a nivel de país entre IDH, predominio de la educación superior pública y políticas para lograr un desarrollo sostenible? ¿Qué alternativas de políticas de educación superior mejorarían el IDH y el desarrollo sostenible a nivel de país, región y mundialmente? ¿Con qué políticas podremos construir los mejores escenarios? ¿Cuales nos conducirán a los peores escenarios? ¿No sería conveniente crear un proyecto como el de Salman Khan (la escuela al revés), donde son los alumnos los que dirigen y desarrollan sus conocimientos guiados por el profesor en el ámbito universitario, dando un paso más allá de los MOOC'S y de la educación superior virtual tradicional? La situación que prevalezca en el ámbito geopolítico, será esencial al respecto.

\section{Recomendaciones}

1. Partir del criterio de que la educación superior es un servicio público. Independiente de cuál sea su fuente de financiamiento. Esto implica que las IES, tanto públicas como privadas, asuman un compromiso público con la sociedad en que están insertas.

2. El acceso ha de ser "igual para todos, en función de los méritos" respectivos tal y como señala en su artículo 26.I la Declaración Universal de los Derechos Humanos.

3. La universidad debe ser un centro de pensamiento crítico, como "una especie de poder intelectual que la sociedad necesita para que la ayude a reflexionar, comprender y actuar", como plantea el Informe Delors.

4. Debe imperar la dimensión ética. Esto implica no supeditar los valores de la cultura de paz, democracia, desarrollo sostenible, equidad, solidaridad, y justicia, a fines 
bélicos o de mercado que atenten contra la condición humana. Adoptar políticas que construyan equidad y autonomía y que impidan la corrupción académica.

5. La universidad ha de aplicar capacidad prospectiva y anticipatoria que le permita, al iluminar posibles alternativas y escenarios, construir la mejor opción posible de futuro. Existen hoy dos tipos de dinámicas: por un lado, la de los mercados, tanto de nuevos proveedores con ánimo de lucro en el ámbito educativo, donde la realidad del mercado tiende a imponerse; como la de acuerdos comerciales en discusión que tienen el apoyo de gobiernos que se beneficiarían. Por otra parte, diversos países y asociaciones del mundo académico han formulado críticas con relación a los efectos de estas tendencias a la mercantilización de la educación superior, qué afectarían en especial a los países en desarrollo por su mayor vulnerabilidad. Lo más probable es que en el corto plazo se combinen ambos escenarios. La regionalización pudiera, a nuestro juicio, constituir una adecuada respuesta a la globalización lucrativa de la educación superior, enfatizando su condición de bien público como se observa en Europa en el proceso de Bolonia. ¿Va en esta dirección el Espacio Europeo de ES (Bologna)?

6. Los Sistemas de Educación Superior en ALC han de fortalecer la identidad cultural propia ante la homogeneidad cultural que pudiera imponer un cierto tipo de globalización.

7. Establecer indicadores adecuados de una universidad nacional de investigación que privilegien el desarrollo sostenible y el crecimiento para el bienestar, para dejar de ser rehenes de los indicadores de productivismo impuestos por el mercado y los rankings.

8. Las Redes de ES en ALC están desempeñando un papel importante. Las universidades deben fomentar políticas de asociación con organizaciones nacionales, regionales e internacionales de redes de ES, con el fin de promover el intercambio de estudiantes, profesores e investigadores, de acuerdo a las necesidades nacionales de desarrollo.

9. Si bien la movilidad académica y el intercambio ha sido una característica central de la educación superior, históricamente, es sólo durante los últimos quince años en que este nivel de enseñanza se ha convertido para algunos en una mercancía o en un servicio susceptible de intercambio comercial entre los diferentes países. El término de internacionalización de la educación superior sigue siendo omnicomprensivo. Por otra parte, nuevos conceptos como el de "nuevos proveedores", y "educación superior transfronteriza" adquieren un rápido desarrollo. Estos conceptos tienen un carácter transfronterizo y una dimensión comercial destinadas a la obtención de beneficios de 
la educación superior. En América Latina y el Caribe, el proceso de internacionalización de la educación superior debe ser preservado a través de una adecuada reglamentación y evitar su desplazamiento o sustitución por una internacionalización con ánimo de lucro. Esto significa establecer reglamentos - de común acuerdo con otros países de la región- que tienden a controlar, en función de las necesidades y los intereses nacionales, las ofertas de ES de carácter transnacional.

10. La universidad en ALC debe cumplir la función social que espera la sociedad de ella, no sólo en equidad en el acceso, sino también en lo que se refiere a estar en el estado del arte en conocimientos, informaciones y propuestas de soluciones. Para esto es necesario crear un clima innovador de carácter transdisciplinario. En las dos últimas décadas -y en especial en los países en desarrollo- muchas de las IES emergentes, e incluso algunas de las tradicionales, han perdido el carácter de instituciones y tienden a asemejarse organizaciones del mundo de los negocios. Es el caso de las universidades corporativas que se asemejan a una empresa. Esto implica una pérdida de su función social, ya que la universidad deja de ser un eslabón entre la educación y el desarrollo, entre el individuo y la sociedad. Es importante insistir en la reinstitucionalización de la universidad, o sea, en la recuperación de su sentido social allí donde lo haya perdido, y no sólo en su reorganización o reforma.

11. Hay que garantizar una adecuada relación con el mundo del trabajo, lo que implica adaptar sus planes y programas de estudios a los requerimientos de éste en ALC. En ALC, prevalecen las carreras tradicionales en lugar de las nuevas necesarias con currículo innovador. Por otra parte, la formación permanente a lo largo de toda la vida, implica una formación universitaria que no sea de perfil estrecho, ajustada meramente a las necesidades inmediatas de las empresas, del mundo del trabajo. Se trata, por el contrario, de dar una formación amplia, que combine la especialización con una sólida formación general, que permita a los graduados universitarios ser más útiles a la sociedad y continuarse desarrollando de forma integral a lo largo de toda la vida.

12. La universidad en ALC ha de asumir el reto de contribuir a la educación cívica, esto es, a la formación de ciudadanos conscientes y responsables. La educación superior debe formar a los estudiantes para que participen activamente en la sociedad, para que defiendan los derechos humanos, la democracia, el desarrollo sostenible, la cultura de paz, la tolerancia y la equidad, entre otros valores esenciales. 
13. Los profesores deben ser co-aprendices con sus alumnos y diseñar ambientes de aprendizajes. Deben esforzarse por inculcar en ellos la afición al estudio y los hábitos mentales que incentiven el auto-aprendizaje (aprender a aprender), a fin de que las IES en ALC sean verdaderos "centros de educación permanente para todos durante toda la vida".

14. Es necesario integrar los sistemas nacionales de educación superior, como estrategia clave en la formulación de políticas de educación superior en ALC. El lograr la integración regional de sistemas de educación superior implica, previamente, su adecuada articulación nacional.

15. La autonomía, que permite a la universidad cumplir en forma cabal sus cometidos, debe ir acompañada de la rendición de cuentas a la sociedad y al estado, lo que implica un estilo de rendición social de cuentas que trasciende lo meramente contable. Autonomía es la total libertad que los miembros de una universidad tienen para, dentro de los criterios de calidad explorar todas las áreas del saber y corrientes del pensamiento humano y trasmitirlos a sus estudiantes, como parte del criterio de calidad académica y responsabilidad social. Esto no significa evadir la legislación aplicable a las IES en ALC, eludir su responsabilidad educativa, ni dejar de rendir cuentas ante la sociedad del uso de los recursos públicos puestos a su disposición para cumplir sus tareas académicas.

16. Los procesos de evaluación y acreditación deben facilitar el tránsito de la cultura de la evaluación a una cultura responsable, autónoma, pertinente y eficiente en las IES en $A L C$. Incorporar a los procesos de acreditación a especialistas extranjeros de reconocida valía en evaluación y acreditación, pero regirse por las normas nacionales y no por agencias extranjeras de acreditación. Incorporar a los procesos de acreditación a especialistas extranjeros de reconocida valía en evaluación y acreditación, pero regirse por las normas nacionales y no por agencias extranjeras de acreditación;

17. Asumir en ALC una concepción estratégica de reforma - no de contrarreforma neoliberal, que es la tendencia predominante en muchas IES de la región - y de cambio, en un marco de planificación y dirección de carácter innovador, en las áreas siguientes: institucional, académica, de organización y pedagógica.

18. La ES a nivel mundial y en ALC debería cumplir sus compromisos con los niveles educativos precedentes, ayudando así a mejorar los niveles primarios y secundarios de educación, aplicando distintas formas de cooperación como la investigación educativa 
para mejorar los procedimientos de articulación entre la educación superior y los niveles precedentes; la formación continua de docentes y directivos del sistema educativo básico y medio; la alfabetización y formación continua de adultos de cualquier nivel educativo; la formación continua de personal integrado al sistema económico en diferentes niveles y áreas ocupacionales y como elemento común a todos estos esfuerzos de colaboración, la implantación de las TIC, en especial la informática y la telemática.

19. Los Sistemas de Educación Superior deben articular fórmulas eficaces y que asignen la igualdad de oportunidades para todos los universitarios, independientemente de si acuden a instituciones públicas o privadas, y que permitan un retorno de la inversión educativa realizada con recursos públicos, de tal manera que el estudiante devolviese a la sociedad y a su país, en su totalidad o en parte, los recursos invertidos en su educación una vez concluida su formación inicial e inserto en el mercado laboral. Habría que hacer un cálculo en las universidades públicas de cuánto cuesta la formación de un estudiante en cada carrera. Ese debería ser el importe de la matrícula, al que se aplicaría una escala de reducciones. La primera de acuerdo con la renta familiar; la segunda por su capacidad o currículo, y la tercera de acuerdo a la distancia que se encuentre su vivienda habitual. Esto implicaría que los buenos estudiantes de estratos económicos desfavorecidos podrían tener una exención del 100\% e incluso una beca con un salario adicional. Mientras que los de familias de los estratos acomodados tendrían que pagar el total de la matrícula. Sé que este argumento no es muy popular en las universidades públicas de ALC, sin embargo lo que hacemos es igualitario pero no equitativo y por ende, socialmente injusto.

20. Para hacer efectivas todas las recomendaciones formuladas en los puntos precedentes, es urgente aplicar a los sistemas de ES de ALC la propuesta de la UNESCO en su CMES (1998) y en su Comisión de Seguimiento (2003, 2004) y luego en su CMES de 2009, acerca de la necesidad de diseñar, en los albores del siglo XXI, una educación superior proactiva y dinámica, que demanda para su éxito una política de estado, una estrategia consensuada de largo plazo, que trascienda el ámbito temporal de los gobiernos, un nuevo pacto social, o contrato moral, como lo llama el Informe Delors, donde cada sector interesado comprometa recursos y esfuerzos para hacer realidad las transformaciones.

Es el momento de reconocer que, a pesar de avances y de logros en unas pocas IES de excepción, aún en ALC no hemos sido capaces de alcanzar la cristalización de un nuevo 
modelo de universidad, en el cual la producción de conocimiento predomine, y no su mera transmisión. Una prioridad en este proceso que deberíamos desarrollar, sería la socialización del conocimiento. De este modo, las IES no se limitarían a ser meras instituciones académicas, pues las universidades se convertirían en las líderes del cambio social.

Somos conscientes de que no hay un modelo único de universidad. Hay muchos ejemplos acertados de reformas de universidad y transformaciones que, mediante la adaptación apropiada a las condiciones de ALC, podemos generalizar y aplicar en nuestras universidades.

\section{Referências}

BRUNNER, J. J. Educación Superior Iberoamericana. Santiago: Cinda, 2016.

COSTAS, A. Alternativa al cosmopolitismo y al populismo. El país, Galicia, 27 nov 2016. Negocios. Disponible en: http://www.ub.edu/graap/Articulos/2016-11-27-

Alternativa\%20cosmopolitismo-PA.pdf

DIAS SOBRINHO, J. Universidade fraturada: conhecimento y responsabilidade social. Avaliação, Campinas; Sorocaba, v. 20, n. 3, nov. 2015. Disponible en:

$<$ http://www.scielo.br/readcube/epdf.php?doi=10.1590/S141440772015000300002\&pid=S1414-40772015000300581\&pdf_path=aval/v20n3/1414-4077aval-20-03-00581.pdf\&lang=pt>. Aceso en: 11 jul. 2017.

LOPEZ SEGRERA, F. Educación superior comparada: tendencias mundiales y de América Latina y el Caribe. Avaliação, Campinas; Sorocaba, v. 21, n. 1, mar. 2016. Disponíble en: $<$ http://www.scielo.br/scielo.php?script=sci_arttext\&pid=S1414$40772016000100013 \& \operatorname{lng}=$ pt\&nrm=iso\&tlng=es>. Aceso en: 11 jul. 2017.

MORIN, E. A necessidade de um pensamento complexo. Candido Mendes (org) e Enrique Rodríguez (ed.), Representação e Complexidade. Rio de Janeiro: Garamond, 2003.

PRIGOGINE, I. O Fim das Certezas. São Paulo: Ed. Unesp, 1996.

OCDE. La enseñanza superior en el 2030. Paris, 2008.

UNESCO.UIS. Tertiary education statistics. Montreal, 2017.

WALLERSTEIN, I. La crisis estructural: los imponderables de mediano plazo. 2013. Disponible en: <http://www2.binghamton.edu/fbc/commentaries/spanish.html>. Aceso en: 11 jul. 2017. 